\title{
$r$-Matrix for the Restricted KdV Flows with the Neumann Constraints
}

\author{
Ruguang $\mathrm{ZHOU}$ \\ Department of Mathematics, Xuzhou Normal University, Xuzhou 221009, P.R. China
}

Received December 15, 1996; Accepted November 25, 1997

\begin{abstract}
Under the Neumann constraints, each equation of the KdV hierarchy is decomposed into two finite dimensional systems, including the well-known Neumann model. Like in the case of the Bargmann constraint, the explicit Lax representations are deduced from the adjoint representation of the auxiliary spectral problem. It is shown that the Lax operator satisfies the $r$-matrix relation in the Dirac bracket. Thus, the integrabilities of these resulting systems with the Neumann constraints are obtained.
\end{abstract}

\section{Introduction}

It is well-known that quite a few finite dimensional integrable systems (FDISs) can be obtained from soliton equation by using the nonlinearization method of Lax pairs [1, 2]. Following [3], we call these FDISs the restricted flows, which can be divided into two kinds, one is the Bargmann system, which is a Hamiltonian system; and another is the Neumann system with some constraints [2]. Many approaches have been developed to study the restricted flows. Among them is the $r$-matrix method. $r$-Matrix formula contains almost necessary information of the FDISs. In this paper, we develop the $r$-matrix method applicable for Neumann systems. We take restricted KdV flows as an example. We first review the results of the restricted KdV flows [1, 3, 何. A hierarchy of FDISs with Neumann constraints are obtained. We focus our attention on the FDISs related with KdV equation, including the well-known Neumann model [5], which describes the motion of a particle on an $(N-1)$-sphere submitted to harmonic forces. We obtain their Lax representations from the adjoint representation of the auxiliary linear problem of KdV hierarchy. All the Lax matrices are $2 \times 2$ matrices (not $N \times N$ !). Then in Section 3, we show that the Lax operator $L(\lambda)$ satisfies the $r$-matrix relation in the Dirac bracket. Finally, by virtue of the general theory of $r$-matrix [6], we obtain the Uhlenbeck motion integrals [7] and prove the involution property in the constrained space (sphere bundle). 


\section{The restricted $\mathrm{KdV}$ flows and their Lax representations}

It is well-known that the $\mathrm{KdV}$ hierarchy associates the following Schrödinger spectral problem

$$
\left(\begin{array}{l}
\psi_{1} \\
\psi_{2}
\end{array}\right)_{x}=U(u, \lambda)\left(\begin{array}{l}
\psi_{1} \\
\psi_{2}
\end{array}\right), \quad U(\lambda, u)=\left(\begin{array}{cc}
0 & 1 \\
-\lambda-u & 0
\end{array}\right) .
$$

To deduce the KdV hierarchy, following [8], we first consider the adjoint representation of (11)

$$
V_{x}=[U, V] \equiv U V-V U
$$

with

$$
V=\left(\begin{array}{cc}
a & b \\
c & -a
\end{array}\right)=\sum_{m=0}^{\infty} V_{m} \lambda^{-m}=\sum_{m=0}^{\infty}\left(\begin{array}{cc}
a_{m} & b_{m} \\
c_{m} & -a_{m}
\end{array}\right) \lambda^{-m}
$$

which leads to

$$
\begin{array}{ll}
b_{i+1}=\mathcal{L} b_{i}, & \mathcal{L}=-\frac{1}{4} \partial^{2}-u+\frac{1}{2} \partial^{-1} u_{x}, \\
a_{i}=-\frac{1}{2} b_{i x}, & c_{i x}=-2 a_{i+1}-2 a_{i} u .
\end{array}
$$

The first few terms are

$$
\begin{aligned}
& a_{0}=b_{0}=0, \quad c_{0}=-1, \quad a_{0}=0, \quad b_{1}=1, \quad c_{1}=-\frac{1}{2} u \\
& a_{2}=\frac{1}{4} u_{x}, \quad b_{2}=-\frac{1}{2} u, \quad c_{2}=\frac{1}{8}\left(u_{x x}+u^{2}\right), \quad b_{3}=\frac{1}{8}\left(u_{x x}+3 u^{2}\right),
\end{aligned}
$$

where $\partial=\frac{\partial}{\partial x}, \partial \partial^{-1}=\partial^{-1} \partial=1$.

Then, we can take the following auxiliary spectral problem

$$
\left(\begin{array}{l}
\psi_{1} \\
\psi_{2}
\end{array}\right)_{t_{n}}=V^{(n)}(\lambda, u)\left(\begin{array}{l}
\psi_{1} \\
\psi_{2}
\end{array}\right)
$$

where

$$
V^{(n)}(\lambda, u)=\sum_{m=0}^{n+1} V_{m} \lambda^{n+1-m}+\left(\begin{array}{cc}
0 & 0 \\
b_{n+2} & 0
\end{array}\right) .
$$

The compatible condition of (1) and (9) yields the zero-curvature equation

$$
U_{t_{n}}-V_{x}^{(n)}+\left[U, V^{(n)}\right]=0, \quad n=1,2, \ldots,
$$

which gives the KdV hierarchy

$$
u_{t_{n}}=-2 b_{n+2, x} .
$$

For $n=0, V^{(0)}=U(u, \lambda)$. 
For $n=1$ we get

$$
V^{(1)}=\left(\begin{array}{cc}
\frac{1}{4} u_{x} & \lambda-\frac{1}{2} u \\
-\lambda^{2}-\frac{u}{2} \lambda+\frac{u_{x x}}{4}+\frac{u^{2}}{2} & -\frac{1}{4} u_{x}
\end{array}\right)
$$

and the $\mathrm{KdV}$ equation ( $\operatorname{set} t_{1}=t$ )

$$
u_{t}=-\frac{1}{4}\left(u_{x x x}+6 u u_{x}\right)
$$

Now we turn to the nonlinearization of Lax pair for the KdV hierarchy. Following [1], we take $N$ distinct $\lambda_{j}^{\prime} s$ and consider the following system

$$
\psi_{1 j, x}=\psi_{2 j}, \quad \psi_{2 j, x}=\left(-\lambda_{j}-u\right) \psi_{1 j}, \quad j=1, \ldots, N .
$$

It is easy to show that (up to a constant factor)

$$
\frac{\delta \lambda_{j}}{\delta u}=\psi_{1 j}^{2}, \quad \mathcal{L} \psi_{1 j}^{2}=\lambda_{j} \psi_{1 j}
$$

As usual [1], we can consider the constraints

$$
b_{k}=\sum_{j=1}^{N} \frac{\delta \lambda_{j}}{\delta u}, \quad k=1,2, \ldots
$$

In this paper we are interested in the Neumann constraint

$$
b_{1}=\sum_{j=1}^{N} \frac{\delta \lambda_{j}}{\delta u}
$$

i.e.,

$$
\langle q, q\rangle=1 .
$$

Here and hereafter, we use the notation: $q_{j}=\psi_{1 j}, p_{j}=\psi_{2 j}, q=\left(q_{1}, \ldots, q_{N}\right)^{T}, p=$ $\left(p_{1}, \ldots, p_{N}\right)^{T}$ and $\langle\cdot, \cdot\rangle$ denotes the stand inner product in space $\mathbb{R}^{N}$.

From (14) and (18) we get

$$
u=\langle p, p\rangle-\langle\Lambda q, q\rangle .
$$

Under this constraint, (14) is nonlinearized into the following system

$$
\left\{\begin{array}{l}
q_{x}=p, \\
p_{x}=-\Lambda q-(\langle p, p\rangle-\langle\Lambda q, q\rangle) q, \\
\langle q, q\rangle=1, \quad\langle q, p\rangle=0 .
\end{array}\right.
$$

When $N=3$, we have the case studied by Neumann [5]. For convenience, we still call (20) the Neumann model.

Making use of the relation

$$
\mathcal{L} \frac{\delta \lambda_{j}}{\delta u}=\lambda_{j} \frac{\delta \lambda_{j}}{\delta u}
$$


we have

$$
\tilde{b}_{i}=\left\langle\Lambda^{i-1} q, q\right\rangle, \quad \tilde{a}_{i}=-\left\langle\Lambda^{i-1} q, p\right\rangle, \quad \tilde{c}_{i}=\left\langle\Lambda^{i-1} p, p\right\rangle, \quad i=2,3, \ldots .
$$

Here and hereafter, symbol tilde means the corresponding nonlinearized quantity and

$$
\tilde{V}=\left(\begin{array}{cc}
-\sum_{k=1}^{N} \frac{p_{k} q_{k}}{\lambda-\lambda_{k}} & \sum_{k=1}^{N} \frac{q_{k}^{2}}{\lambda-\lambda_{k}} \\
-1-\sum_{k=1}^{N} \frac{p_{k}^{2}}{\lambda-\lambda_{k}} & \sum_{k=1}^{N} \frac{p_{k} q_{k}}{\lambda-\lambda_{k}}
\end{array}\right) .
$$

Under the condition (19), the following equation still holds

$$
\tilde{V}_{x}=[\tilde{U}, \tilde{V}] .
$$

Conversely a direct calculation can verify that (23) is just the Lax representation of equation (20). For simplicity of notation, we set $L(\lambda)=-\tilde{V}$, and $M_{i}=\tilde{V}^{(i)}$. Therefore we have

Theorem 1. The equation (20) has the Lax representation

$$
L_{x}=\left[M_{0}, L\right]
$$

with

$$
L(\lambda)=\left(\begin{array}{cc}
A(\lambda) & B(\lambda) \\
C(\lambda) & -A(\lambda)
\end{array}\right), \quad M_{0}(\lambda)=\left(\begin{array}{cc}
0 & 1 \\
-\lambda+\langle\Lambda q, q\rangle-\langle p, p\rangle & 0
\end{array}\right),
$$

where

$$
A(\lambda)=\sum_{k=1}^{N} \frac{p_{k} q_{k}}{\lambda-\lambda_{k}}, \quad B(\lambda)=-\sum_{k=1}^{N} \frac{q_{k}^{2}}{\lambda-\lambda_{k}}, \quad C(\lambda)=1+\sum_{k=1}^{N} \frac{p_{k}^{2}}{\lambda-\lambda_{k}} .
$$

In the same way, we can discuss the nonlinearizations of the auxiliary spectral problem

$$
\left(\begin{array}{l}
\psi_{1 j} \\
\psi_{2 j}
\end{array}\right)_{t_{n}}=V^{(n)}\left(\lambda_{j}, u\right)\left(\begin{array}{l}
\psi_{1 j} \\
\psi_{2 j}
\end{array}\right), \quad j=1, \ldots, N .
$$

under the constraints (19) and (20) and obtain a hierarchy of finite dimensional systems with the Neumann constraint $\langle q, q\rangle=1$. We call this the restricted $t_{n}$-flow. Just like in the case of the Bargmann constraint, the identity

$$
V_{t_{n}}=\left[V^{(n)}, V\right]
$$

is a nonlinearization of the Lax representation of the restricted $t_{n}$-flow. In particular, for $n=1$ we obtain the following system

$$
\left\{\begin{array}{l}
q_{t}=\Lambda p-\langle\Lambda q, p\rangle q+\frac{1}{2}\langle p, p\rangle p+\frac{1}{2}\langle\Lambda q, q\rangle p \\
p_{t}=-\Lambda^{2} q-\frac{1}{2}\langle p, p\rangle \Lambda q+\frac{1}{2}\langle\Lambda q, q\rangle \Lambda q-\langle\Lambda p, p\rangle q \\
\quad+\left\langle\Lambda^{2} q, q\right\rangle q-\frac{1}{2}\langle\Lambda q, q\rangle^{2} q+\frac{1}{2}\langle p, p\rangle^{2} q+\langle\Lambda q, p\rangle p \\
\langle q, q\rangle=1
\end{array}\right.
$$


and the Lax representation

Theorem 2. The equation (29) possesses the following Lax representation

$$
\begin{aligned}
& L_{x}=\left[M_{1}, L\right], \\
& M_{1}(\lambda)=\left(\begin{array}{cc}
-\langle\Lambda q, p\rangle & \lambda-\frac{1}{2}(\langle p, p\rangle-\langle\Lambda q, q\rangle) \\
D(\lambda) & \langle\Lambda q, p\rangle
\end{array}\right),
\end{aligned}
$$

where

$$
D(\lambda)=-\lambda^{2}-\frac{\langle p, p\rangle-\langle\Lambda q, q\rangle}{2} \lambda-\langle\Lambda p, p\rangle+\left\langle\Lambda^{2} q, q\right\rangle-\frac{\langle\Lambda q, q\rangle^{2}-\langle p, p\rangle^{2}}{2} .
$$

Remark. After a simple calculation, we can show that: if $(q, p)^{T}$ satisfies the system of equation (20) and (29), then $u=\langle p, p\rangle-\langle\Lambda q, q\rangle$ solve the $\mathrm{KdV}$ equation (13). Besides from [2], we know it must be a finite-band solution of the KdV equation. Thus we can obtain the finite-band solution through solving the two finite dimensional systems with the Neumann constraints.

\section{$3 \quad r$-Matrix for the restricted flow with Neumann constraints}

We know that the standard Poisson bracket for the two smooth functions $f(p, q), g(p, q)$ in the symplectic space $\mathbb{R}^{2 N}, \sum_{j=1}^{N} d p_{j} \wedge d q_{j}$ is defined by

$$
\{f, g\}=\sum_{i=1}^{N}\left(\frac{\partial f}{\partial q_{i}} \frac{\partial g}{\partial p_{i}}-\frac{\partial f}{\partial p_{i}} \frac{\partial g}{\partial q_{i}}\right) .
$$

Since we are dealing with a constrained system with the holonomos constraints

$$
F \equiv\langle q, q\rangle-1=0, \quad G \equiv\langle q, p\rangle=0,
$$

i.e., the constraint manifold is the sphere bundle $T S^{N-1}$

$$
T S^{N-1}=\left\{(q, p) \in \mathbb{R}^{2 N} \mid\langle q, q\rangle=1,\langle q, p\rangle=0\right\}
$$

, we shall use the Dirac bracket [9], instead of the standard Poisson bracket.

The Dirac bracket for the constraint (14) is [9]

$$
\{f, g\}_{D}=\{f, g\}+\frac{1}{2}\{f, F\}\{G, g\}-\frac{1}{2}\{f, G\}\{F, g\} .
$$

A direct calculation yields

$$
\left\{q_{k}, q_{l}\right\}_{D}=0, \quad\left\{q_{k}, p_{l}\right\}_{D}=\delta_{k l}-q_{k} q_{l}, \quad\left\{p_{k}, p_{l}\right\}_{D}=-q_{k} p_{l}+p_{k} q_{l} .
$$

Proposition 1. The Neumann systems (20) and (29) can be written as the following nonstandard Hamiltonian forms

$$
q_{x}=\left\{q, H_{1}\right\}_{D}, \quad p_{x}=\left\{p, H_{1}\right\}_{D}
$$


and

$$
q_{x}=\left\{q, H_{2}\right\}_{D}, \quad p_{x}=\left\{p, H_{2}\right\}_{D},
$$

respectively. Here

$$
H_{1}=\frac{1}{2}(\langle p, p\rangle+\langle\Lambda q, q\rangle)
$$

and

$$
H_{2}=\frac{1}{2}\left\langle\Lambda^{2} q, q\right\rangle+\frac{1}{2}\langle\Lambda p, p\rangle+\frac{1}{4}\langle\Lambda q, q\rangle\langle p, p\rangle-\frac{1}{8}\langle p, p\rangle^{2}-\frac{1}{8}\langle\Lambda q, q\rangle^{2} .
$$

It is easy to show that

$$
\begin{aligned}
& \{A(\lambda), A(\mu)\}_{D}=\{B(\lambda), B(\mu)\}_{D}=0, \\
& \{C(\lambda), C(\mu)\}_{D}=4(A(\lambda)-A(\mu))+4(A(\mu) C(\lambda)-A(\lambda) C(\mu)), \\
& \{A(\lambda), B(\mu)\}_{D}=\frac{2}{\lambda-\mu}(B(\lambda)-B(\mu))-2 B(\lambda) B(\mu), \\
& \{A(\lambda), C(\mu)\}_{D}=\frac{2}{\mu-\lambda}(C(\lambda)-C(\mu))+2 B(\lambda) C(\mu)-2 B(\lambda), \\
& \{B(\lambda), C(\mu)\}_{D}=\frac{4}{\mu-\lambda}(A(\mu)-A(\lambda))-4 B(\lambda) A(\mu),
\end{aligned}
$$

where $\lambda, \mu$ are two arbitrary parameters.

We make use of the familiar notation [11]: $L_{1}(\lambda)=L(\lambda) \otimes I, L_{2}(\mu)=I \otimes L(\mu)$, where $I$ is the unit $2 \times 2$ matrix; and set

$$
\left.\left\{L_{1}(\lambda), L_{(} \mu\right)\right\}_{D}^{j k, m n}=\left\{L(\lambda)^{j m}, L(\mu)^{k n}\right\}_{D} .
$$

It follows from (41)-(45) that

Theorem 3. The Lax operator $L(\lambda)$ admits the following $r$-matrix representation

$$
\left\{L_{1}(\lambda), L_{2}(\mu)\right\}_{D}=\left[r_{12}(\lambda, \mu), L_{1}(\lambda)\right]-\left[r_{21}(\lambda, \mu), L_{2}(\mu)\right]
$$

with

$$
\begin{aligned}
& r_{12}(\lambda, \mu)=\frac{2}{\mu-\lambda} P+2 S+Q_{12}, \\
& r_{21}(\lambda, \mu)=-\frac{2}{\mu-\lambda} P+2 S+Q_{21},
\end{aligned}
$$

where

$$
P=\left(\begin{array}{llll}
1 & 0 & 0 & 0 \\
0 & 0 & 1 & 0 \\
0 & 1 & 0 & 0 \\
0 & 0 & 0 & 1
\end{array}\right), \quad S=\left(\begin{array}{ll}
0 & 0 \\
1 & 0
\end{array}\right) \otimes\left(\begin{array}{ll}
0 & 0 \\
1 & 0
\end{array}\right),
$$




$$
\begin{aligned}
Q_{12} & =\left(\begin{array}{ll}
1 & 0 \\
0 & 0
\end{array}\right) \otimes\left(\begin{array}{cc}
B(\mu) & 0 \\
-2 A(\mu) & -B(\mu)
\end{array}\right)+\left(\begin{array}{cc}
0 & 0 \\
1 & 0
\end{array}\right) \otimes\left(\begin{array}{cc}
0 & B(\mu) \\
-C(\mu) & 0
\end{array}\right), \\
Q_{21} & =\left(\begin{array}{cc}
B(\lambda) & 0 \\
-2 A(\lambda) & -B(\lambda)
\end{array}\right) \otimes\left(\begin{array}{ll}
1 & 0 \\
0 & 0
\end{array}\right)+\left(\begin{array}{cc}
0 & B(\lambda) \\
-C(\lambda) & 0
\end{array}\right) \otimes\left(\begin{array}{ll}
0 & 0 \\
1 & 0
\end{array}\right) .
\end{aligned}
$$

In order to show that $r_{12}$ is an $r$-matrix, we must check that the bracket (24) is skewsymmetric and satisfies the Jacobi identity. The skew-symmetry property follows from the relation:

$$
Q_{12}=-\left[M, L_{2}(\mu)\right], \quad Q_{21}(\lambda)=\left[M, L_{1}(\lambda)\right] \quad \text { and } \quad\left[\left[M, L_{2}\right], L_{1}\right]=\left[\left[M, L_{1}\right], L_{2}\right],
$$

where

$$
M=\left(\begin{array}{cccc}
0 & 0 & 0 & 0 \\
1 & 0 & 0 & 0 \\
-1 & 0 & 0 & 0 \\
0 & 0 & 0 & 0
\end{array}\right)
$$

Through some lengthy but straightforward calculations, we can obtain the Jacobi identity

$$
\left[\bar{L}_{1},\left[r^{12}, r^{13}\right]+\left[r^{12}, r^{23}\right]\right]+\left[\bar{L}_{1},\left\{L_{2}, r^{13}\right\}_{D}-\left\{\bar{L}_{3}, r^{12}\right\}_{D}\right]+\text { cycle perm. }=0,
$$

where all quantities are understood to $\mathcal{G} \otimes \mathcal{G} \otimes \mathcal{G}, \mathcal{G}=\operatorname{sl}(2) \otimes C\left(\lambda, \lambda^{-1}\right)$; and as usual the superscript denotes the space which the corresponding tensor acts on nontrivially,

$$
\bar{L}_{1}=L \otimes I \otimes I, \quad r^{12}=r_{12} \otimes_{I}, \quad \text { etc. }
$$

An immediate consequent [6] of (24) is that

$$
\left\{L_{1}^{2}(\lambda), L_{2}^{2}(\mu)\right\}_{D}=\left[\bar{r}_{12}(\lambda, \mu), L_{1}(\lambda)\right]-\left[\bar{r}_{21}(\lambda, \mu), L_{2}(\mu)\right]
$$

with

$$
\bar{r}_{i j}(\lambda, \mu)=\sum_{k=0}^{1} \sum_{l=0}^{1} L_{1}^{1-k}(\lambda) L_{2}^{1-l}(\mu) r_{i j}(\lambda, \mu) L_{1}^{k}(\lambda) L_{2}^{l}(\mu)
$$

Then it follows from (31) we have

$$
\left\{\operatorname{Tr} L_{1}^{2}(\lambda), \operatorname{Tr} L_{2}^{2}(\mu)\right\}_{D}=\frac{1}{4} \operatorname{Tr}\left\{L^{2}(\lambda), L^{2}(\mu)\right\}_{D}=0
$$

which ensures the involution property of the integrals of motion obtained from expanding $L^{2}(\lambda)$ in powers of $\lambda$.

For our Lax pair $L(\lambda)$, we have

$$
-\frac{1}{2} \operatorname{Tr} L^{2}(\lambda)=-A^{2}(\lambda)-B(\lambda) C(\lambda)=\sum_{k=1}^{N} \frac{q_{k}^{2}}{\lambda-\lambda_{k}}+\sum_{k=1}^{N} \frac{1}{\lambda-\lambda_{k}} \sum_{l=1}^{N^{\prime}} \frac{\left(q_{k} p_{l}-p_{k} q_{l}\right)^{2}}{\lambda_{k}-\lambda_{l}}
$$

Here and hereafter, $\sum_{l=1}^{N^{\prime}} \ldots$ denotes the index of sum, which is not equal to $k$. It follows that

$$
J_{k}=q_{k}^{2}+\sum_{l=1}^{N^{\prime}} \frac{\left(q_{k} p_{l}-p_{k} q_{l}\right)^{2}}{\lambda_{k}-\lambda_{l}}, \quad k=1,2, \ldots, N
$$


are $N$ involution systems, which are just the conserved quantities obtained by Moser and Uhlenbeck [7]. Therefore, it follows from (33) that

$$
\left\{J_{k}, J_{l}\right\}_{D}=0, \quad k, l=1,2, \ldots, N .
$$

As $\sum_{k=1}^{N} J_{k}=\langle q, q\rangle=1$, we know only $N-1 J_{k}{ }^{\prime} s$ are functionally independent among $J_{1}, J_{2}, \ldots, J_{N}$.

Set

$$
F_{k}=\sum_{j=1}^{N} \lambda_{j}^{k} J_{j}
$$

then $F_{k}$ and $J_{i}$ are in involution.

Noticing

$$
H_{0}=\frac{1}{2} F_{1}
$$

and

$$
H_{1}=\frac{1}{2} F_{2}-\frac{1}{8} F_{1}^{2}
$$

we know that both the system (20) and (29) is completely integrable.

\section{Conclusion}

In this paper, we obtained the Lax representations and the $r$-matrix for the restricted KdV flows with the Neumann constraints. This approach can also be applied to other finite dimensional integrable systems with the Neumann constraints. Besides, for all the restricted $\mathrm{KdV}$ flows which were discussed, we can perform the separation of variables to integrate them following [10]; thus obtain the finite-band solution of the KdV hierarchy.

\section{Acknowledgement}

I am very grateful to Professors Gu Chaohao, Hu Hesheng and Cao Cewen for their guidance and also thanks Professor Zhou Zixiang and Professor Qiao Zhijun for some valuable discussions.

This project is supported by The Doctoral Programme Foundation of Institute High Education and by the Nature Science Foundation of Education Committee of Jiangsu province.

\section{References}

[1] Cao C.W., Science in China A, 1990, V.7, 701-707.

[2] Cao C.W. and Geng X.G., Classical Integrable Systems Generated through the Nonlinearization of Eigenvalue Problems, in: Proc. Conf. on Nonlinear Physics, Shanghai 1989, Research reports in Physics, Berlin-Springer, 1990, 66-78. 
[3] Rauch-Wojciechowski S., Phys. Letts A, 1991, V.160, 241-246.

[4] Zeng Y.B. and Li Y.S., J. Phys. A, 1993, V.26, L273-L278.

[5] Neumann C., Crelle Journal, 1859, V.56, 46.

[6] Babelon O. and Viallet C.M., Phys. Lett. B, 1990, V.237, N 3, 411.

[7] Uhlenbeck K., Equivariant Harmonic Maps into Sphere, in: Proc. of Tulannne Conf. on Harmonic Maps, Springer lecture notes in Mathematics, 946, 1982, 146.

[8] Tu G.Z., J. Phys. A: Math. Gen., 1989, V.22, N 13, 2375-2392.

[9] Sundermeyer K., Constrianed Dynamics with Applications to Yang-Mills Theory, General Relatiovity, Classical Spin, Dual String Model, Lectures notes in Physics 169, Springer-Verlag, 1982.

[10] Harnad J. and Winternitz P., Commun. Math. Phys., 1995, V.172, 263-285.

[11] Faddeev L.D. and Takhtajian L.A., Hamiltonian Methods in the Theory of Solitons, Springer-Verlag, 1987. 